\title{
Photonics-Advances in Fundamental Sciences and Engineering Technologies of Light
}

\author{
Nelson Tansu \\ Founding Editor-in-Chief of Photonics, Center for Photonics and Nanoelectronics, Department of \\ Electrical and Computer Engineering, Lehigh University, Bethlehem, PA 18015, USA; \\ E-Mail: Tansu@Lehigh.Edu; Tel.: +1-610-758-2678
}

Received: 18 March 2014 / Accepted: 19 March 2014 / Published: 20 March 2014

Photonics is a field of sciences that focuses on the pursuit of the understanding basic properties of light, the interaction of light with materials, the fundamental concepts and technologies for generating and controlling the properties of light, the concept and technologies for transmitting and signal processing of light, the engineering of these technologies for manipulating light applicable for systems implementation. The optical light covers the electromagnetic radiation from X-ray, deep ultraviolet, ultraviolet, visible, infrared, and terahertz spectral regimes. Optical sciences is one of the earliest and most important areas of study in physical sciences. The successful understanding of wave optics within the Maxwell's electromagnetism theory has resulted in the development of physical optics [1,2], and its impact has penetrated areas in other disciplines including chemical and biological sciences, engineering and technology, and medical sciences. The birth of quantum theory emanated from the understanding of the discrete energy of light by Planck and Einstein, and understanding of discrete energy levels in atoms was also attributed to understanding the interaction of light and matter. Quantum electrodynamics and quantum optics formed the basis of the advanced theory of light-matter interaction. Today, fundamental concepts from photonics and optics have been widely implemented in technologies addressing substantial challenges in such fields as communications, medical and health sciences, energy efficiency and renewable energy, homeland security and defense, environmental and sustainable living, among others.

Advances in photonics and optics encompass both experimental and theoretical findings in basic sciences, technology, and engineering of light. The advances in both disruptive and progressive technologies in photonics and optics have enabled the development of new technologies which have transformed our life and addressed significant challenges in society. The journal Photonics (ISSN 23046732) provides a platform to share knowledge, being an online open access journal covering both the fundamental theory and applications of optical sciences and photonics. Photonics strives to provide a means for authors to disseminate their scientific findings-both theoretical/simulations and 
experimental works - in highly accessible peer-reviewed publications, in order to achieve a fast dissemination of high impact works within the scientific community. Several key advances in the photonics and optical sciences and engineering are listed below.

New optical imaging and optical coherence tomography methods in biomedical optics [3-6] have resulted in low cost and practical methods to detect cancer cells at an early stage. The development of new optical imaging and holography methods has also improved medical imaging technology [7]. Recent developments in optogenetics [8] have also provided a new technique using both optics and genetics to control and modify the responses of neurons in cells.

Developments in semiconductor epitaxy, materials, and new device concepts based on compound semiconductors, specifically III-V and III-Nitride, have revolutionized the progress in telecommunication lasers and solid state lighting technologies [9-22]. Further, the improved understanding in the fundamental properties of quantum-based active regions, with regard to nanostructures formed by III-V and III-Nitride semiconductors, has enabled the disruptive advance resulting in low-threshold laser diodes for telecommunication and display, and high efficiency light-emitting diodes for solid state lighting, respectively. Recent developments of X-ray free electron lasers, deep UV emitters, visible LEDs and lasers, infrared lasers, mid and far IR lasers, and terahertz lasers have received tremendous attention in recent years. Significant progress has also been achieved in the development of new device architectures for improved efficiency in solar cells, faster and more responsive photodetector technologies, and higher speed and lower cost optical modulators.

Research in the physics of semiconductors and nanostructures has enabled further progressive improvements of these devices which have resulted in practical technologies currently being implemented in our daily life. As an example, advances in material synthesis and epitaxy have resulted in the ability to analyze materials composition to atomic precision levels, resulting in the ability to modify and control the optical and optoelectronic properties in a transformational manner. Nanostructure engineering of the active regions has been implemented to achieve high efficiency LEDs and low threshold lasers. New quantum confined structures based on quantum cascade lasers have also been realized, and these works have resulted in progress towards achieving lasers emitting in the mid-IR up to terahertz spectral ranges. Tremendous potential exists in new materials, device concepts, and applications in semiconductor photonics and optoelectronics which are currently being created in new directions impacting energy, healthcare, communications, water purifications, and environments.

Advances in nanofabrication technology have resulted in the ability to form sub-wavelength structures for controlling the propagation of light waves in media. One of the most successful examples in the field of nanophotonics is related to the development of photonic crystal technologies [23-28], which have been widely implemented for controlling the photonic bandgap, enabling compact photonic integrated circuits, new optical device functionalities, and many other new applications. Recent progress has also focused on the development of nanophotonic structures based on sub-wavelength gratings [29,30] and self-assembled colloidal based arrays [31,32]. The pursuit-both in fundamental theory and application - of cavity optomechanics in nano electromechanical structures (NEMS) is also an exciting research area [33,34].

Advancements in the ability to form high quality quantum dot active media [35-38] have also provided new device functionalities for solar cells, lasers, and light emitting diodes. The integration of 
quantum dot and nanophotonic technologies have recently shown promise in its future potential for enabling a practical solid-state based quantum computing device platform [39,40].

Plasmonics technologies and metamaterials have been among the most active research topics in the fields of optical sciences and engineering [41-47]. The new functionalities enabled by plasmonics based technologies are at the early stage with innovations only limited by the need for new ideas and creativities. The exciting field of plasmonics has provided many new directions in the field of optics.

One of the mainstays of photonics and optical sciences and engineering has been the devices and systems developed for fulfilling the requirements of optical communications [48,49]. The increasing demand in this regard has not been met by similar investments in development of new technologies. The innovations in low cost device technologies based on photonic integrated circuits [50-54] to fulfill the system-driven requirements in optical communication will remain an important area of research topics in optics. Nonlinear optics and ultrafast optics [55-59] remain exciting areas of research that could result in new fundamental concepts, new phenomena, and new device functionalities. Portable and compact terahertz sources had also been reported by using difference frequency generation [60].

The progress in computing power has escalated the importance of computational photonics, computational nanostructures, and physics and simulation of materials and optoelectronic devices [61-67]. The expensive experiments can be reduced or planned with lower iteration rate by having the improved computational models of materials, nanostructures, and device concepts. Advances in computational models of physical systems have already enabled very accurate prediction of electronics and optical properties of materials, nanostructures, devices, and integrated photonics devices.

Low cost silicon based photonics, graphene photonics, and flexible electronics/optoelectronics materials and devices [68-75] have tremendous potential for enabling low-cost sensors and medical therapeutic devices. Recent research in these fields have been very active, and progress has demonstrated the potential of these platforms for enabling their practical implementation.

The idea of splitting water via of the use of catalyst by using solar radiation to produce hydrogen fuel has been suggested and pursued for sometimes [76-78]. The key challenges in practical implementation of this concept require solutions that provide absorber materials appropriate for tandem cell designs, and structures with stable chemical properties under illuminated aqueous environment. The pursuit of appropriate materials and nanostructures will enable efficient conversion efficient for solar hydrogen, which has tremendous impacts for renewable energy and energy storage technologies.

Understanding of photon-electron-phonon interaction in nanostructures and materials still needs to be developed [79]. The rich new areas of fundamental research in this discipline will have tremendous impact in the thermal management, heat transfer, laser cooling, and thermoelectric areas.

In summary, the fields of photonics and optics have contributed tremendously in the development of new technologies resulting in transformational impact on society. Technologies anchored in the fundamental and innovative applications in photonics and optics have been integrated in daily life with transformational impacts in wide range of fields such as health care, communications, energy, environments, and homeland security. The progress in new material syntheses, engineered material designs, device structures, and systems implementations in photonics and optical sciences and engineering will continue to provide disruptive advances and progressive solutions for addressing key challenges in society. 


\section{Acknowledgments}

The author acknowledges the support of the New Century Endowed Chair Professorship Fund at Lehigh University, and US National Science Foundation (ECCS 1028490, ECCS 1408051, and DMR 0907260).

\section{Conflicts of Interest}

The author declares no conflict of interest.

\section{References}

1. Maxwell, J.C. A dynamical theory of the electromagnetic field. Phil. Trans. R. Soc. Lond. 1865, doi:10.1098/rstl.1865.0008.

2. Born, M.; Wolf, E. Principles of Optics; Cambridge University Press: Cambridge, UK, 2002.

3. Liang, X.; Graf, B.W.; Boppart, S.A. Imaging engineered tissues using structural and functional optical coherence tomography. J. Biophotonics. 2009, 2, 643-655.

4. Linninger, A.A. Biomedical systems research-New perspectives opened by quantitative medical imaging. Comput. Chem. Eng. 2012, 36, 1-9.

5. Svanberg, S. Biophotonics-techniques and applications. Laser Photon. Rev. 2013, 7, A43-A44.

6. West, J.L.; Halas, N.J. Engineered nanomaterials for biophotonics applications: Improving sensing, imaging, and therapeutics. Annu. Rev. Biomed. Eng. 2003, 5, 285-292.

7. Boustany, N.N.; Boppart, S.A.; Backman, V.; Microscopic imaging and spectroscopy with scattered light. Annu. Rev. Biomed. Eng. 2010, 12, 285-314.

8. Deisseroth, K. Optogenetics. Nat. Method. 2011, 8, 26-29.

9. Coleman, J.J.; Young, J.D.; Garg, A. Semiconductor quantum dot lasers: A tutorial. J. Lightwave. Tech. 2011, 29, 499-510.

10. Vurgaftman, I.; Bewley, W.W.; Canedy, C.L.; Kim, C.S.; Kim, M.; Lindle, J.R.; Merritt, C.D.; Abell, J.; Meyer, J.R. Mid-IR type-II interband cascade lasers. IEEE J. Sel. Top. Quant. 2011, 17, 1435-1444.

11. Tansu, N.; Yeh, J.Y.; Mawst, L.J. Physics and characteristics of 1200-nm InGaAs and 1300-1400 $\mathrm{nm}$ InGaAsN quantum-well lasers by metalorganic chemical vapor deposition. J. Phys. Condens. Matter Phys. 2004, 16, S3277-S3318.

12. Bank, S.R.; Bae, H.; Goddard, L.L.; Yuen, H.B.; Wistey, M.A.; Kudrawiec, R.; Harris, J.S. Recent progress on $1.55 \mu \mathrm{m}$ dilute-nitride lasers. IEEE J. Quantum Elect. 2007, 43, 773-785.

13. Reece, P.J.; Jagadish, C. Semiconductor nanostructure optoelectronics. Mater. Sci. En. B Adv. 2012, 177, 695-695.

14. Beling, A.; Campbell, J.C. InP-based high-speed photodetectors. J. Lightwave. Technol. 2009, 27, 343-355.

15. Feezell, D.F.; Speck, J.S.; DenBaars, S.P.; Nakamura, S. Semipolar (2021) InGaN/GaN light-emitting diodes for high-efficiency solid-state lighting. J. Display Technol. 2013, 9, 190-198.

16. Wierer, J.J.; Tsao, J.Y.; Sizov, D.S. Comparison between blue lasers and light-emitting diodes for future solid-state lighting. Laser Photon. Rev. 2013, 7, 963-993. 
17. Zhao, H.P.; Liu, G.Y.; Zhang, J.; Poplawsky, J.D.; Dierolf, V.; Tansu, N. Approaches for high internal quantum efficiency green InGaN light-emitting diodes with large overlap quantum wells. Opt. Express 2011, 19, A991-A1007.

18. Zhang, J.; Tansu, N. Engineering of AlGaN-Delta-GaN quantum wells gain media for mid- and deep-ultraviolet lasers. IEEE Photon. J. 2013, 5, 2600209, doi:10.1109/JPHOT.2013.2248705.

19. Faist, J.; Capasso, F.; Sivco, D.L.; Sirtori, C.; Hutchinson, A.L.; Cho, A.Y. Quantum cascade laser. Science 1994, 264, 553-556.

20. Kumar, S.; Hu, Q.; Reno, J.L. $186 \mathrm{~K}$ operation of terahertz quantum-cascade lasers based on a diagonal design. Appl. Phys. Lett. 2009, 94, 131105, doi:10.1063/1.3114418.

21. Butler, J.K.; Ackley, D.E.; Botez, D. Coupled-mode analysis of phase-locked injection laser arrays. Appl. Phys. Lett. 1984, 44, 293-295.

22. Kanskar, M.; Earles, T.; Goodnough, T.J.; Stiers, E.; Botez, D.; Mawst, L.J. 73\% CW power conversion efficiency at $50 \mathrm{~W}$ from $970 \mathrm{~nm}$ diode laser bars. Electron. Lett. 2005, 41, 245-247.

23. Yablonovitch, E. Inhibited spontaneous emission in solid-state physics and electronics. Phys. Rev. Lett. 1995, 340, 841-844.

24. John, S. Strong localization of photons in certain disordered dielectric superlattices. Phys. Rev. Lett. 1987, 58, 2486-2489.

25. Yablonovitch, E.; Gmitter, T.J. Photonic band structure: The face-centered-cubic case. Phys. Rev. Lett. 1989, 63, doi:10.1103/PhysRevLett.63.1950.

26. Johnson, S.G.; Villeneuve, P.R.; Fan, S.H.; Joannopoulos, J.D. Linear waveguides in photonic-crystal slabs. Phys. Rev. B 2000, 62, 8212-8222.

27. Norris, D.J. Photonic crystals: A view of the future. Nat. Mater. 2007, 6, 177-178.

28. Mohseni, P.K.; Kim, S.H.; Zhao, X.; Balasundaram, K.; Kim, J.D.; Pan, L.; Rogers, J.A.; Coleman, J.J.; Li, X. GaAs pillar array-based light emitting diode fabricated by metal-assisted chemical etching. J. Appl. Phys. 2013, 114, 064909, doi:10.1063/1.4817424.

29. Chang-Hasnain, C.J.; Yang, W. High-contrast gratings for integrated optoelectronics. Adv. Optic. Photon. 2012, 4, 379-440.

30. Karagodsky, V.; Sedgwick, F.G.; Chang-Hasnain, C.J. Theoretical analysis of subwavelength high contrast grating reflectors. Optic. Express 2010, 18, 16973-16988.

31. Li, X.H.; Song, R.B.; Ee, Y.K.; Kumnorkaew, P.; Gilchrist, J.F.; Tansu, N. Light extraction efficiency and radiation patterns of III-Nitride light-emitting diodes with colloidal microlens arrays with various aspect ratios. IEEE Photon. J. 2011, 3, 489-499.

32. Koo, W.H.; Youn, W.; Zhu, P.F.; Li, X.H.; Tansu, N.; So, F. Light extraction of organic light emitting diodes using defective hexagonal-close-packed array. Adv. Function. Mater. 2012, 22, 3454-3459.

33. Lin, Q.; Rosenberg, J.; Chang, D.; Camacho, R.; Eichenfield, M.; Vahala, K.J.; Painter, O. Coherent mixing of mechanical excitations in nano-optomechanical structures. Nat. Photon. 2010, 4, 236244.

34. Poot, M.; Tang, H. Broadband nanoelectromechanical phase shifting of light on a chip. Appl. Phys. Lett. 2014, 104, doi:10.1063/1.4864257.

35. Grundmann, M.; Stier, O.; Bimberg, D. InAs/GaAs pyramidal quantum dots: Strain distribution, optical phonons, and electronic structure. Phys. Rev. B 1995, 52, 11969-11981. 
36. Liu, G.T.; Stintz, A.; Li, H.; Malloy, K.J.; Lester, L.F. Extremely low room-temperature threshold current density diode lasers using InAs dots in In $0.15 \mathrm{Ga} 0.85$ As quantum well. Electron. Lett. 1999, 35, 1163-1165.

37. Gu, T.; El-Emawy, M.A.; Yang, K.; Stintz, A.; Lester, L.F. Resistance to edge recombination in GaAs-based dots-in-a-well solar cells. Appl. Phys. Lett. 2009, 95, doi:10.1063/1.3277149.

38. Huffaker, D.L.; Park, G.; Zou, Z.; Shchekin, O.B.; Deppe, D.G. $1.3 \mu \mathrm{m}$ room-temperature GaAs-based quantum-dot laser. Appl. Phys. Lett. 1998, 73, doi:10.1063/1.122534.

39. Santori, C.; Fattal, D.; Vuckovic, J.; Solomon, G.S.; Yamamoto, Y. Indistinguishable photons from a single-photon device. Nature 2002, 419, 594-597.

40. Buckley, S.; Rivoire, K.; Vuckovic, J. Engineered quantum dot single-photon sources. Rep. Prog. Phys. 2012, 75, doi:10.1088/0034-4885/75/12/126503.

41. Hess, J.B.; Pendry, S.A.; Maier, R.F.; Oulton, J.M.; Hamm, K.L. Active nanoplasmonic metamaterials. Nat. Mater. 2012, 11, 573-584.

42. Soukoulis, C.M.; Wegener, M. Past achievements and future challenges in the development of three-dimensional photonic metamaterials. Nat. Photon. 2011, 5, 523-530.

43. Kadic, M.; Buckmann, T.; Schittny, R.; Wegener, M. Metamaterials beyond electromagnetism. Rep. Prog. Phys. 2013, 76, doi:10.1088/0034-4885/76/12/126501.

44. Bilotti, F.; Sevgi, L. Metamaterials: Definitions, properties, applications, and FDTD-based modeling and simulation. Int. J. RF Microw. CAE 2012, 22, 422-438.

45. Naik, G.V.; Shalaev, V.M.; Boltasseva, A. Alternative plasmonic materials: Beyond gold and silver. Adv. Mater. 2013, 25, 3264-3294.

46. Atwater, H.A.; Polman, A. Plasmonics for improved photovoltaic devices. Nat. Mater. 2010, 9 , 205-213.

47. Gan, Q.; Bartoli, F.J.; Kafafi, Z.H. Plasmonic-enhanced organic photovoltaics: Breaking the 10\% efficiency barrier. Adv. Mater. 2013, 25, 2385-2396.

48. Kaminow, I.; Li, T.; Willner, A.E. Optical Fiber Telecommunications Volume VIA: Components and Subsystems; Academic Press: San Diego, CA, USA, 2013.

49. Kaminow, I.; Li, T.; Willner, A.E. Optical Fiber Telecommunications Volume VIB: Systems and Networks; Academic Press: San Diego, CA, USA, 2013.

50. Koch, T.L.; Koren, U. Semiconductor photonics integrated circuits. IEEE J. Quantum Electron. 1991, 27, 641-653.

51. Kish, F.A.; Welch, D.; Nagarajan, R.; Pleumeekers, J.L.; Lal, V.; Ziari, M.; Nilsson, A.; Kato, M.; Murthy, S.; Evans, P.; et al. Current status of large-scale InP photonic integrated circuits. IEEE J. Sel. Top. Quantum Electron. 2011, 17, 1470-1489.

52. Coldren, L.A. Monolithic tunable diode lasers. IEEE J. Sel. Top. Quantum Electron. 2000, 6, $988-$ 999.

53. Koch, T.L. III-V and Silicon Photonic Integrated Circuit Technologies. In Proceedings of the Optical Fiber Communication Conference, Los Angeles, CA, USA, 4-8 March 2012; paper OTh4D.1.

54. Dai, D.; Bauters, J.; Bowers, A.J.E. Passive technologies for future large-scale photonic integrated circuits on silicon: polarization handling, light non-reciprocity and loss reduction. Light Sci. Appl. 2012, 1, e1, doi:10.1038/1sa.2012.1. 
55. Weiner, A.M. Ultrafast optical pulse shaping: A tutorial review. Opt. Commun. 2011, 284, 3669-3692.

56. Weiner, A.M. Ultrafast Optics; Wiley: Hoboken, NJ, USA, 2009.

57. Dudley, J.M.; Taylor, J.R. Ten years of nonlinear optics in photonic crystal fibre. Nat. Photon. 2009, 3, 85-90.

58. Boyd, R.W. Slow and fast light: Fundamentals and applications. J. Modern Optic. 2009, 56, $18-19$.

59. Boyd, R.W. Nonlinear Optics; Academic Press: San Diego, CA, USA, 2003.

60. Zhao, P.; Ragam, S.; Ding, Y.J.; Zotova, I.B. Compact and portable terahertz source by mixing two frequencies generated simultaneously by a single solid-state laser. Opt. Lett. 2010, 35, 3979-3981.

61. Taflove, A.; Johnson, S.G.; Oskooi, A. Advances in FDTD Computational Electrodynamics: Photonics and Nanotechnology; Artech House: Norwood, MA, USA, 2013.

62. Liu, V.; Miller, D.A.B.; Fan, S.H. Highly tailored computational electromagnetics methods for nanophotonic design and discovery. Proc. IEEE 2013, 101, 484-493.

63. Van de Walle, C.G.; Janotti, A. Advances in electronic structure methods for defects and impurities in solids. Phys. Status Solid. B 2011, 248, 19-27.

64. Tan, C.K.; Zhang, J.; Li, X.H.; Liu, G.Y.; Tayo, B.O.; Tansu, N. First-principle electronic properties of dilute-As GaNAs alloy for visible light emitters. J. Display Technol. 2013, 9, 272-279.

65. Lany, S.; Zunger, A. Assessment of correction methods for the band-gap problem and for finite-size effects in supercell defect calculations: Case studies for $\mathrm{ZnO}$ and GaAs. Phys. Rev. B 2008, 78, doi:10.1103/PhysRevB.78.235104.

66. Li, S.; Fu, Y. 3D TCAD Simulation for Semiconductor Processes, Devices and Optoelectronics; Springer: Berlin, Germany, 2012.

67. Liu, G.Y.; Zhang, J.; Tan, C.K.; Tansu, N. Efficiency-droop suppression by using large-bandgap AlGaInN thin barrier layers in InGaN quantum wells light-emitting diodes. IEEE Photon. J. 2013, 2, 2201011, doi:10.1109/JPHOT.2013.2255028.

68. Pavesi, L.; Vivien, L. Handbook of Silicon Photonics; Taylor \& Francis: Boca Raton, FL, USA, 2013.

69. Jalali, B.; Fathpour, S. Silicon photonics. J. Lightwave Technol. 2006, 24, 4600-4615.

70. Soref, R. The past, present, and future of silicon photonics. IEEE J. Sel. Top. Quantum Electron. 2006, 12, 1678-1687.

71. Foster, M.A.; Turner, A.C.; Sharping, J.E.; Schmidt, B.S.; Lipson, M.; Gaeta, A.L. Broad-band optical parametric gain on a silicon photonic chip. Nature 2006, 441, 960-963.

72. Avouris, P. Graphene: Electronic and photonic properties and devices. Nano Lett. 2010, 10, 4285-4294.

73. Grigorenko, N.; Polini, M.; Novoselov, K.S. Graphene plasmonics. Nat. Photon. 2012, 6, 749-758.

74. Sun, Y.; Rogers, J.A. Inorganic semiconductors for flexible electronics. Adv. Mater. 2007, 19, 1897-1916. 
75. Reuss, R.H.; Chalamala, B.R.; Moussessian, A.; Kane, Michael G.; Kumar, A.; Zhang, D.C.; Rogers, J.A.; Hatalis, M.; Temple, D.; Moddel, G.; Eliasson, B.J.; Estes, M.J.; et al. Macroelectronics: Perspectives on technology and applications. Proc. IEEE 2005, 93, 1239-1256.

76. Hu, S.; Xiang, C.; Haussener, S.; Berger, A.D.; Lewis, N.S. An analysis of the optimal band gaps of light absorbers in integrated tandem photoelectrochemical water-splitting systems. Energy Environ. Sci. 2013, 6, 2984-2993.

77. Walter, M.G.; Warren, E.L.; McKone, J.R.; Boettcher, S.W.; Mi, Q.; Santori, E.A.; Lewis, N.S. Solar water splitting cells. Chem. Rev.2010, 110, 6446-6473.

78. Phivilay, S.P.; Roberts, C.A.; Puretzky, A.A.; Domen, K.; Wachs, I.E. Fundamental Bulk/surface structure photoactivity relationships of supported (Rh2-yCryO3)/GaN photocatalysts. J. Phys. Chem. Lett. 2013, 4, 3719-3724.

79. Chen, G. Nanoscale Energy Transport and Conversion: A Parallel Treatment of Electrons, Molecules, Phonons, and Photons; Oxford University Press: Oxford, UK, 2005.

(C) 2014 by the authors; licensee MDPI, Basel, Switzerland. This article is an open access article distributed under the terms and conditions of the Creative Commons Attribution license (http://creativecommons.org/licenses/by/3.0/). 